\title{
Web-based Visual Analytics of Lifestyle Data in MyHealthAvatar
}

\author{
Youbing Zhao, Farzad Parvinzamir, Xia Zhao, Zhikun Deng, Nikolaos Ersotelos, Feng Dong, \\ Gordon Clapworthy \\ Department of Computer Science and Technology, University of Bedfordshire \\ Luton, UK \\ +44-1582-743717
}

\{youbing.zhao, farzad.parvinzamir, xia.zhao, zhikun.deng, nikolaos.ersotelos, feng.dong, gordon.clapworthy\}@beds.ac.uk

\begin{abstract}
MyHealthAvatar is a project designed to collect lifestyle and health data to promote citizen's wellbeing. As a lifetime companion of citizens the amount of data to be collected is large. It is almost impossible for citizens, patients and doctors to view, utilise and understand these data without proper visual presentation and user interaction. Visual analytics of lifestyle data is one of the key features of MyHealthAvatar. This paper presents the visual analytics components in MyHealthAvatar to facilitate health and lifestyle data presentation and analysis, including 3D avatar, dashboard, diary, timeline, clock view and map. These components can be used cooperatively to achieve flexible visual analysis of spatial temporal lifestyle and health data.
\end{abstract}

\section{Categories and Subject Descriptors}

H.3.5 [Web-based services]

\section{General Terms}

Design, Experimentation, Human Factors

\section{Keywords}

MyHealthAvatar, Visual Analytics, lifestyle data, 3d Avatar, Dashboard, Diary, Timeline, ClockView, Map

\section{INTRODUCTION}

The MyHealthAvatar project [1] is a research project through which the feasibility of an innovative representation of the health status of citizens for future healthcare - 4D MyHealthAvatar, is studied. A 4D avatar is a unique interface that allows data access, collection, sharing and analysis by utilising modern ICT technology, overcoming the shortcomings of the existing resources in Europe, which are highly fragmented. It will become the citizen's lifelong companion, providing long-term and consistent health status information of the individual citizen along a timeline representing the citizen's life, starting from birth. Data sharing is encouraged, which potentially provides an extensive collection of population data and offers extremely valuable support to clinical research. The system is equipped with tools to facilitate data analysis and knowledge discovery.

MyHealthAvatar believes that healthcare should not only care for patients but also look after the health and wellbeing of all citizens. It needs to be available to healthy people by maintenance of a healthy lifestyle and the notification of early symptoms. Hence, MyHealthAvatar targets both healthy citizens and patients.

MyHealthAvatar offers significant assistance to users by:

- displaying related information in a body-centric view around the avatar.

- allowing simulation via access to the model repositories, supported by the computing resource that is provided by the architecture.

- $\quad$ performing visually assisted data analysis (i.e. visual analytics) to extract clinically meaningful information from the heterogeneous data of individual/shared avatars, such as the patterns of symptoms, experience of treatments, medicines, selfcare guidelines, risk factors etc..

As a proof of concept, MyHealthAvatar project is building a web-based system myhealthavatar.org [2], with the following features:

A. Information Collection and Access.

The system has:

- Internal data repositories to store individual data for the avatars;

- An internal model repository to store models commonly used by all the avatars;

- Links to external sources, such as hospitals' Electronic Health Records (EHRs) and other data and model repositories.

Ranges of technologies are used in data collection and information access, including:

Information extraction from the web and data collection using mobile apps; 
Semantics and linked data to support the data/model searching and reasoning.

\section{B. Data Management and Sharing}

MyHealthAvatar is a tool that allows highly self-motivated data management and user-centred data collection, supported by the necessary data integrity measures. The citizens have full rights to share their own data. Individual citizens decide how the data is shared by stakeholders. For example, the avatar data can be shared with physicians for clinical treatment, with research organisations to support clinical research, or with other individual patients on a volunteer basis. These options are supported by the underlying ICT architecture, which has adequate measures to ensure data reliability and integrity.

\section{Information Analysis and Visual Analysis}

The purpose of health and medical information collection is to promote healthy lifestyles of citizens and to assist clinical decision making. Information analysis is a vehicle by which citizens can make basic analysis based on knowledge from the platform and medical professionals can augment their clinical knowledge with heterogeneous information from the avatar for clinical decision-making and knowledge exploration.

As a project focusing on health and lifestyle data collection, access, analysis and sharing, without proper visualisation, it is not possible for the user to select, view, understand and gain knowledge from a large collection of data. Thus, visualisation and visual analysis is a critical part for the effective uitilisation of data collected and stored on the MyHealthAvatar platform. This paper focuses on the visual analysis components and is organised as follows: section II briefly introduces related fitness tracking devices; section III introduces data supported by MyHealthAvatar; section IV presents the main visual analytics components in MyHealthAvatar and section IV discusses the future work.

\section{RELATED WORK}

With increasing popularity of fitness and health sensors and apps in recent years, such as Fitbit [3], Withings [4], iHealth [5], Moves [6], etc., there has been increasing interests in building centralised health and fitness data repository. Google Health was a personal health information centralisation service introduced by Google in 2008 but discontinued in 2011 [7]. HealthVault [8] from Microsoft integrated a number of fitness sensors on the market to provide a central repository and APIs for data access, but its emphasis is on data storage and does not provide powerful visualisation tools for data analysis. Fluxtream [9] is an open source project to provide data storage of fitness sensors and basic visualisation tools, but unlike MyHealthAvatar it does not support connections to medical systems thus the visualisation only focuses on fitness data.

\section{SENSORS AND DATA}

MyHealthAvatar data are health and lifestyle oriented and can be categorised into health sensor data and medical data. As the medical data is managed by medical partners in the project and is imported from the hospital information system, such as Optima, they are not the focus of this paper. Here we are more interested in health data and lifestyle, including data from mobile apps.

\subsection{Sensors and Apps}

In recent years, there has been a boom in the health sensor market. Sensors evolved from traditional devices such as the step meter to internet-enabled devices such as Fitbit [3], Withings [4], iHealth [5], etc. They measure steps, walking distance, calories, sleep quality, heart rate, weight, etc., based on the device model. Some devices measure more medical oriented data, such as blood pressure, glucose, etc. Meanwhile, with the rise of smart phones, fitness mobile apps also become an important data source of health and lifestyle data. Mobile phones with proper sensors installed are capable of not only measuring the step number with an accelerometer but also recording the location of the user with a GPS sensor, thus keeping track of both the fitness data and daily lifestyle data of the user.

Moves [6] is a very popular app for fitness and lifestyle recording. Moves automatically records the step number and location of the user. It also calculates calories burned and distance of movement. It automatically recognise the activity type, such as walking, running, cycling, transport, etc. You can either view the distance, duration, steps, and calories data on the mobile phone or export the data from the Moves server. An automatic daily storyline with time and location are recorded and shown on the map in the Moves app.

Currently MyHealthAvatar supports importing data from a user's Fitbit, Withings and Moves accounts via APIs provided by these devices. More devices and apps will be supported in the future.

\subsection{Human Input Data}

In addition to data automatically collected by fitness and lifestyle sensors and apps, there are data that may need manual input such as events, food, etc. Manual inputting is a very important data source, which can be used not only for data input but also for data editing and error fixing. Consequently, MyHealthAvatar supports human input data in addition to sensor data and medical data. The medical data themselves are composed of data from medical devices as well as manual input from doctors.

\section{VISUAL ANALYSIS COMPONENTS}

MyHealthAvatar provides several web-based components for visualisation and analysis of personal health and lifestyle data, including 3D avatar, dashboard, timeline, diary, clockview and map.

Lifestyle, health, fitness and medical data are inherently time dependent. To visualise time-varying data, linear and radial layouts can used. A timeline is a linear visualisation of data spanning a long period while the clockview uses the common clock metaphor to visualise daily events in a radial layout. A calendar is another common layout to visualise human life related events. To visualise spatiotemporal data, MyHealthAvatar uses the map and integrates the clock view with the map. A calendar like diary is also provided for activity data input, edit and planning.

\subsection{D Avatar}

A web-based interactive $3 \mathrm{D}$ avatar is proposed as an interface to support health and medical information display. Related information can be visualised in a body-centric view around the avatar, which offers significant assistance to patients and doctors. Consequently, web-based interactive visualisation of the whole 
body anatomy is the key for body related information visualisation.

The benefits of using a 3D human body model rather than $2 \mathrm{D}$ pictures in an avatar web interface include the followings:

1. 3D human model is more realistic and intuitive.

2. A 3D human model is a natural representation for an avatar.

3. With user interactions, a 3D human model can represent and gather more information than $2 \mathrm{D}$ pictures do.

4. The user or patient can learn better about his or her health conditions and disease as well as human anatomy.

Figure 1 is an example view of the 3D avatar.

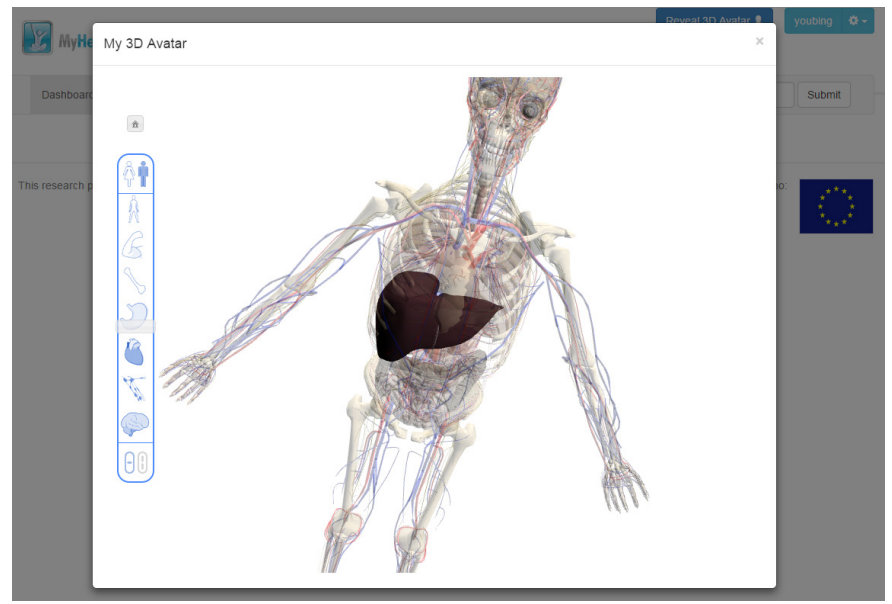

Figure 1 3D avatar example screenshot

\subsection{Dashboard}

There are many components and data sources available through the web-based MyHealthAvatar portal. However, it may be difficult for a user to grasp an overview with the most important health status information. To present the user a quick overview of their health status, MyHealthAvatar provides a dashboard as the user's front page. The dashboard provides a summary of the user's latest health status with important notifications. It may include several simple visualisation components to depict data for a recent period. Figure 2 shows the example dashboard with data tiles, a map and a timeline.

\subsection{Diary}

MyHealthAvatar provides health data collection, storage and access to citizens, patients and doctors. The data can either be automatically collected or manually input. For lifestyle and health tracking, the data are often time dependant, especially date dependant. A traditional form of date-based data organisation, display and editing is a calendar. In MyHealthAvatar, a calendarbased diary is used for daily data display as well as event input, editing and planning. Figure 3 is an example view of MyHealthAvatar calendar with the event editor. The calendar displays a brief summary of the fitness data such as daily steps, walking and transportation distance as well as calories burned. The data come from Fitbit, Withings and Moves.

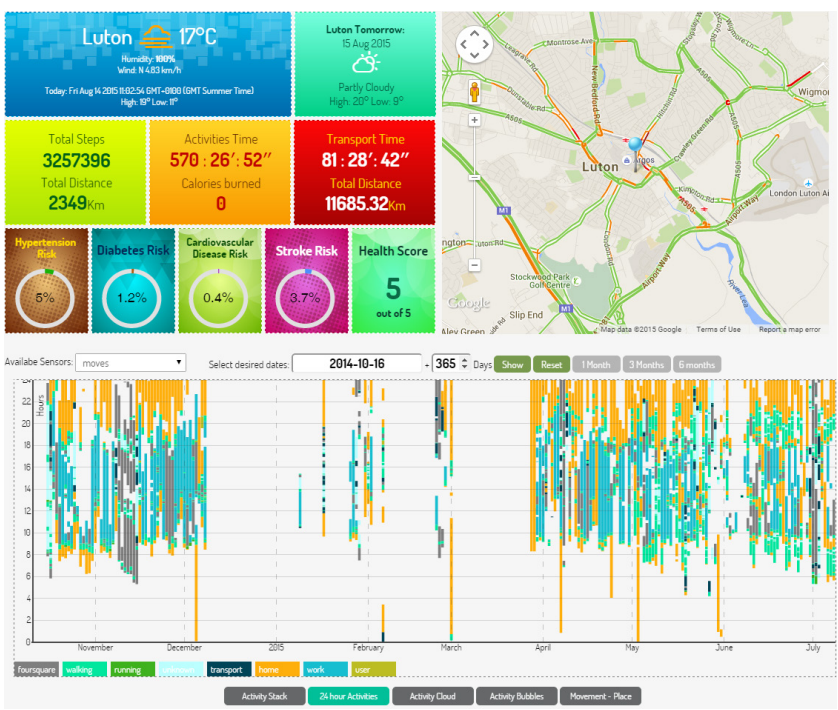

Figure 2 Example screenshot of dashboard
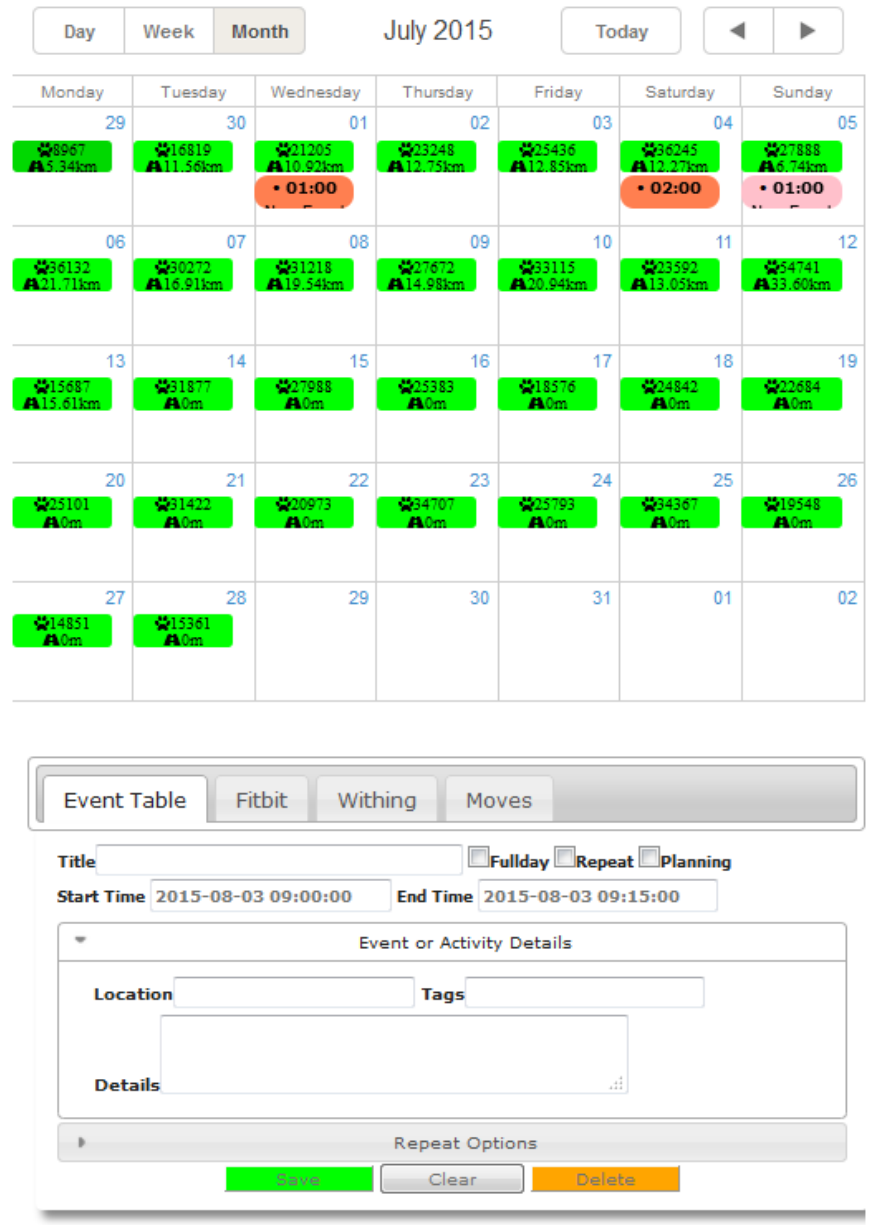

Figure 3 An example view of the diary and event editor 


\subsection{Timeline}

A timeline is a traditional method to visualise time-varying data and events in a linear layout. Compared to a calendar, a timeline is more suitable for visualising continuous variables that cover a relatively long period, such as health indicators and medical measurements. Activity events, which are time dependent, can also be shown in a timeline if a longer time scale is. In addition to interactive time range selections, the timeline supports interactive filtering and automatic clustering of events if the number of events is large. Figure 4 is an example of daily activity events visualised in a timeline.

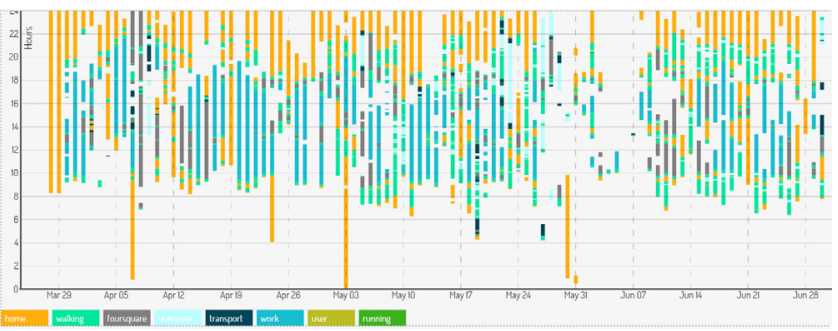

Figure 4 A timeline view of daily events

\subsection{ClockView}

For daily activities, timeline provides visualisation over a relatively long period. Interactive timelines provide zooming to smaller scales. However, the linear layout may make it difficult for the user to understand and compare periodic events at a daily basis. A fine-grained view of activities within one day is better visualised in a radial layout. A natural real-life way of radial daily time representation is the clock. MyHealthAvatar uses a similar radial layout called ClockView to visualise daily events. Activity types are marked by icons and colours. When the user hovers the mouse over icons and arcs more detailed information will be displayed, as shown in Figure 5.

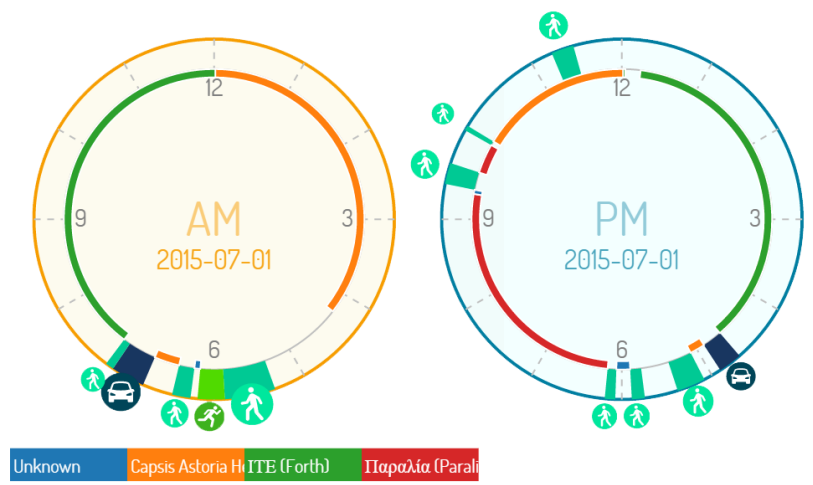

Figure 5 ClockView example

\subsection{Map}

While dairy, timeline and ClockView are majorly designed for visual analysis of temporal data, they can hardly be used to visualise spatial data and location data. The use of map is to make a natural and intuitive spatiotemporal visualisation and analysis of the user's locations and routes to facilitate analysis, understanding and knowledge discovery of the lifestyle. The map implementation is based on Google Maps [11]. Currently it is used for visualisation and analysis of the Moves data but is capable of supporting other location sensor based Apps. Figure 6

is the map visualisation of Moves daily data using line segments and heatmap.

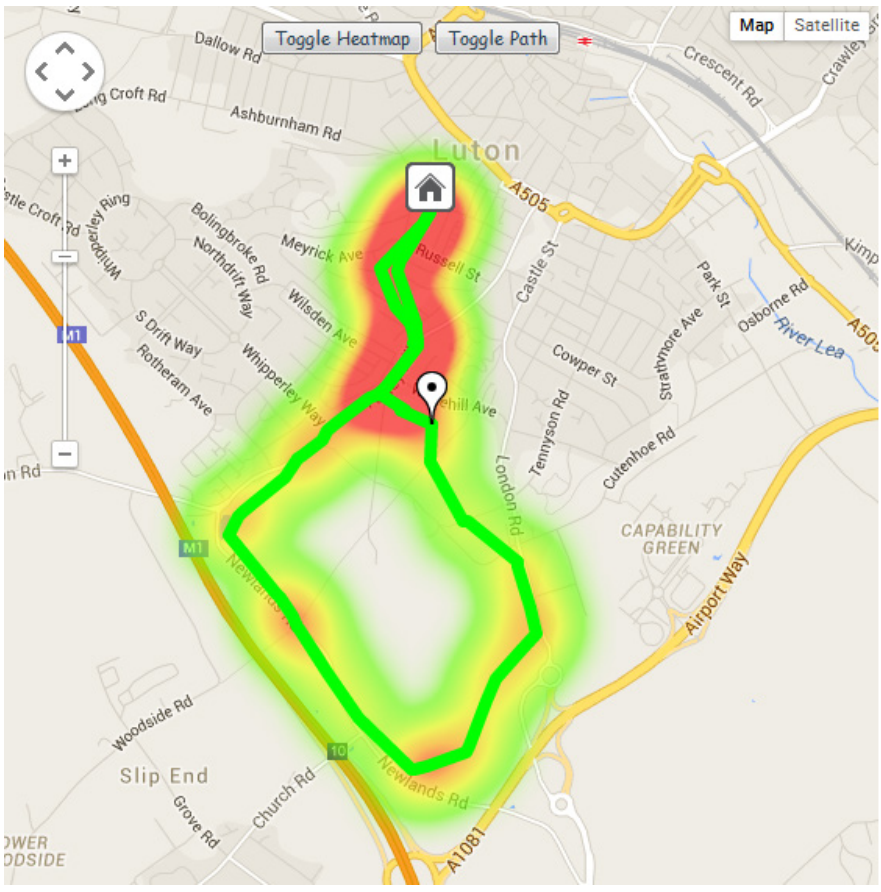

Figure 6 Map visualisation of routes and locations of daily activities from Moves

In addition, MyHealthAvatar uses a compound view composed of diary, map and clock view to visualise daily event data, as shown in Figure 7. The advantage of the compound view is that it provides integrated spatiotemporal visualisation and analysis. The page itself provides the user a view of multiple data sources and the user does not need to refer to multiple pages to make analysis of the spatiotemporal data collected and stored on MyHealthAvatar platform.

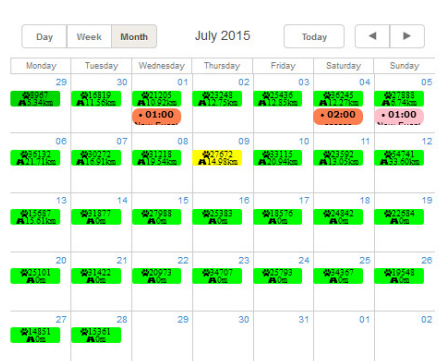

Evert Table Fithit Withing Noves

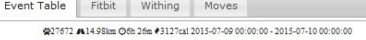

Title

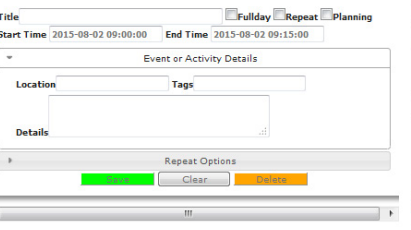

Figure 7 Integrated view of diary, map and clock view

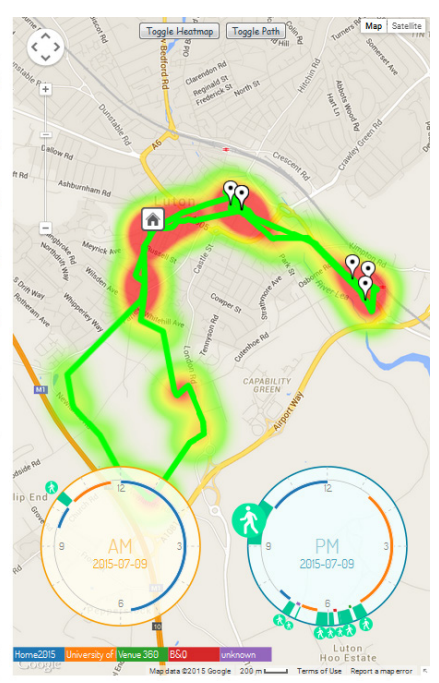




\section{FUTURE WORK}

The current visual analytics work in MyHealthAvatar is only designed for a single user. In the next stage, MyHealthAvatar will focus more on data sharing among multiple users. How to effectively visualise data sharing and shared data will be more of interest. Consequently shared data visualisation and analysis will be the focus in the next stage.

\section{ACKNOWLEDGMENTS}

MyHealthAvatar project is funded by the 7th Framework Programme of European Commission - ICT (FP7-ICT-2011-9). We would like to thank the European Commission for the funding and thank the project officers and reviewers for their indispensible support for the project.

\section{REFERENCES}

[1] MyHealthAvatar Project, http://www.myhealthavatar.eu/
[2] MyHealthAvatar Platform, http://myhealthavatar.org

[3] Fitbit: http://www.fitbit.com/

[4] Withings: http://www.withings.com

[5] iHealth: http://www.ihealthlabs.com/

[6] Moves: https://www.moves-app.com/

[7] Google Health (discontinued) : http://www.google.com/intl/en-GB/health/about/

[8] HealthVault: https://www.healthvault.com/

[9] Fluxstream: https://fluxtream.org/

[10] Youbing Zhao, Xia Zhao, Feng Dong, Gordon Clapworthy, Nikolaos Ersotelos, Enjie Liu, WebGL-based interactive rendering of whole body anatomy for web-oriented visualisation of avatar-centered digital health data. BIBE 2013: 1-4

[11] Google Maps: https://www.google.co.uk/maps 